\title{
GLOBALLY EXACT OPERATOR SPACES
}

\author{
Massoud Amini, Alireza Medghalchi and Hamed Nikpey \\ Tarbiat Modares University, Kharazmi University and Shahid Rajaei \\ University, Iran
}

\begin{abstract}
Exact operator spaces are known to be locally reflexive, but the converse is not true. We introduce the notion of global exactness and show that it is equivalent to reflexivity for injective operator spaces.
\end{abstract}

\section{INTRODUCTION}

Following a discovery of Archbold and Batty ([2]) on the lack of mincontinuity property for certain canonical binormal embeddings of the algebraic tensor product of two non nuclear $C^{*}$-algebras (or their biduals) into the bidual of their min-tensor product, Kirchberg ([15]) introduced the notion of exactness for $C^{*}$-algebras and proved later in [17] that for this larger class (compared to the class of nuclear $C^{*}$-algebras) some of these complications are resolved. Similar min-continuity type conditions are also introduced by Effros and Haagerup in [7] (and some of these notions are already known to be equivalent).

The operator space version of exactness is introduced by Pisier in [19] and studied by Effros, Ozawa and Ruan in [9]. The theory has played a significant role in recent developments in the theory of $C^{*}$-algebras and operator spaces. For more details on the notion of exactness, we refer the reader to Wassermann ([27]) (for $C^{*}$-algebras) and Effros and Ruan ([10]) and Pisier ([20]) (for operator spaces).

Kirchberg showed in [16] that a $C^{*}$-algebra $B$ is exact if and only if $B$ is locally reflexive and $B^{* *}$ is weakly exact. The notion of weak* exactness is introduced by Dong and Ruan in [6] and it is shown that an operator space

2010 Mathematics Subject Classification. 46L07, 47L25.

Key words and phrases. Operator space, injective operator space, injective envelope, exact operator space, globally exact operator space, local reflexivity.

The first author was partly supported by a grant from IPM (No. 90430215). 
$V$ is exact if and only if $V$ is locally reflexive and $V^{* *}$ is weak ${ }^{*}$ exact. Also it is known that an operator space is nuclear if and only if it is exact (or locally reflexive) and has the WEP (weak expectation property), see [9]. The relation between reflexivity and local reflexivity of operator spaces with mincontinuity type properties (operator space analogs of those of Archbold and Batty) are explored in [6] using the results of Effros, Junge and Ruan in [8].

It is known that local reflexivity is in general strictly weaker than exactness both for $C^{*}$-algebras (examples provided by Kirchberg) and operator spaces (the operator space $\left(\ell^{1}(\mathbb{N}), M A X\right)$ is an example). In this paper we introduce the notion of global exactness and show that it is equivalent to reflexivity for injective operator spaces.

Let $H$ be a Hilbert space. A concrete operator space is a subspace $V$ of $B(H)$. For an operator space $V$, the space $M_{n}(V)$ of $n \times n$ matrices with entries in $V$ inherits an operator norm as a subspace of $B\left(H^{n}\right)$. Given operator spaces $V$ and $W$, the $n$-th amplification $\varphi_{n}: M_{n}(V) \rightarrow M_{n}(W)$ of a linear map $\varphi: V \rightarrow W$ is $\varphi_{n}(T)=\left[\varphi\left(T_{i, j}\right)\right]$, for $T=\left[T_{i, j}\right] \in M_{n}(V)$. The completely bounded norm of $\varphi$ is defined by

$$
\|\varphi\|_{c b}=\sup \left\{\left\|\varphi_{n}\right\|: n \in \mathbb{N}\right\} .
$$

A linear map $\varphi$ is called completely bounded (resp., a complete isometry) if $\|\varphi\|_{c b}<\infty$ (respectively, each $\varphi_{n}$ is an isometry). For concrete operator spaces $V \subseteq B(H)$ and $W \subseteq B(K), \varphi$ is called completely positive if each $\varphi_{n}$ is positive with respect to the positive cones of $B\left(H^{n}\right)$ and $B\left(K^{n}\right)$. The operator spaces $V$ and $W$ are completely isometric (resp., completely isomorphic) if there is a surjective complete isometry $\varphi: V \rightarrow W$ (resp., a bijective completely bounded map $\varphi: V \rightarrow W$ with completely bounded inverse). We write $V \cong W$ when $V$ and $W$ are completely isometric.

An abstract operator space is a vector space $V$ with norms on matrix spaces $M_{n}(V)$ such that $V$ is completely isometric to a concrete operator space. We do not distinguish between concrete and abstract operator spaces.

An operator space $V$ is injective if, given operator spaces $W_{1} \subseteq W_{2}$, with completely isometric inclusion, any completely bounded map $\varphi_{1}: W_{1} \rightarrow V$ can be extended to a completely bounded map $\varphi_{2}: W_{2} \rightarrow V$ with $\left\|\varphi_{2}\right\|_{c b}=$ $\left\|\varphi_{1}\right\|_{c b}$. By the celebrated Wittstock Theorem, $B(H)$ is an injective operator space, for any Hilbert space $H([28])$. Hamana $([13,14])$ and Ruan $([24])$ independently showed that for any operator space $V \subseteq B(H)$ there is a minimal injective operator subspace $I(V)$ of $B(H)$ containing $V$, called the injective envelope of $V$.

A $C^{*}$-extension of a unital operator space $V$ is a pair $(A, i)$ consisting of a unital $C^{*}$-algebra $A$, and a unital complete isometry $i: V \rightarrow A$, such that $i(V)$ generates $A$ as a $C^{*}$-algebra. Two $C^{*}$-extensions $(A, i)$ and $(B, j)$ are $V$ equivalent if there exist a $*$-isomorphism $\pi: B \rightarrow A$ such that $\pi \circ j=i$. The 
$C^{*}$-envelope of $V$ is the unique extension $(A, i)$ with the universal property of the next theorem (for more detail see [3, Theorem 4.3.1]).

Theorem 1.1 (Arveson-Hamana). If $V$ is a unital operator space, then there exists a unique (up to $V$-equivalence) $C^{*}$-extension $(A, i)$ of $V$ with the following universal property: Given any $C^{*}$-extension $(B, j)$ of $V$, there exists a unique surjective $*$-homomorphism $\pi: A \rightarrow B$ such that $\pi \circ i=j$.

\section{GLOBAL EXACTNESS AND REFLEXIVITY}

Let $E, F$ be operator spaces. Put

$$
d_{c b}(E, F)=\inf \left\{\|\varphi\|_{c b},\left\|\varphi^{-1}\right\|_{c b}\right\}
$$

where the infimum runs over all complete isomorphisms $\varphi: E \rightarrow F$. If $E, F$ are not completely isomorphic, set $d_{c b}(E, F)=\infty$. A finite dimensional operator space $W$ is $\lambda$-exact if for any $\varepsilon>0$ there exist an integer $n \in \mathbb{N}$ and a subspace $S$ of $M_{n}(\mathbb{C})$ such that $d_{c b}(W, S)<\lambda+\varepsilon$. If $\operatorname{dim} W=n$, then $W$ is $n$-exact $([10$, section 14.2]). Put

$$
d_{e x}(W)=\inf \{\lambda: W \text { is } \lambda \text {-exact }\} .
$$

This could be regarded as the exactness approximation constant of $W$. Clearly, $d_{e x}(W) \leq \operatorname{dim} W$. To make this work for an arbitrary operator space $V$, let

$$
d_{e x}(V)=\sup \left\{d_{e x}(W): W \subseteq V, W \text { is finite dimensional }\right\} .
$$

We say that $V$ is $\lambda$-exact if $d_{e x}(V) \leq \lambda$, and that $V$ is exact if it is 1-exact. For more details, see $[23,10]$.

Definition 2.1. An operator space $V$ is called globally exact if any finite dimensional operator subspace $W \subseteq V$ is completely isometric to a subspace of $M_{n}(\mathbb{C})$, for some positive integer $n$.

Clearly each globally exact operator space is exact, and each subspace of a globally exact operator space is again globally exact. Also, a finite direct sum $\bigoplus_{i=1}^{n} V_{i}$ of operator spaces is globally exact if and only if each factor $V_{i}$ is globally exact.

THEOREM 2.2. For an operator space $V$, the following are equivalent:

(i) $V$ is globally exact;

(ii) For any finite dimensional operator subspace $W \subseteq V, I(W)$ is finite dimensional.

Proof. Let $V$ be globally exact and $W \subseteq V$ be any finite dimensional operator subspace. Then there is $n \in \mathbb{N}$ and a subspace $S$ of $M_{n}(\mathbb{C})$ such that $W \cong S \subseteq M_{n}(\mathbb{C})$. Thus $I(W) \cong I(S) \subseteq M_{n}(\mathbb{C})$ is finite dimensional.

Conversely, let (ii) holds and $W$ be any finite dimensional operator subspace of $V$. By assumption, $I(W)$ is finite dimensional, and so is completely 
isometrically isomorphic to $p A p^{\perp}$, for some finite dimensional $C^{*}$-algebra $A$ and a projection $p \in A([25])$. As $A \cong \bigoplus_{i=1}^{m} M_{n_{i}}(\mathbb{C})$, for some positive integers $m, n_{i}$, there are positive integers $p_{i}, q_{i}$ such that

$$
W \subseteq I(W) \cong p A p^{\perp} \cong \bigoplus_{i=1}^{m} M_{p_{i}, q_{i}}(\mathbb{C})
$$

Thus there is an operator subspace $S \subseteq \bigoplus_{i=1}^{m} M_{p_{i}, q_{i}}(\mathbb{C})$ such that $W \cong S$. This means that $V$ is globally exact.

Let $V \subseteq B(H)$ be an operator space. The Paulsen operator system $\mathcal{S}(V)$ is defined by

$$
\mathcal{S}(V)=\left[\begin{array}{cc}
\mathbb{C} I_{H} & V \\
V^{*} & \mathbb{C} I_{H}
\end{array}\right]=\left\{\left[\begin{array}{cc}
\lambda & T \\
S^{*} & \mu
\end{array}\right]: T, S \in V, \lambda, \mu \in \mathbb{C}\right\}
$$

in $\mathbb{M}_{2}(B(H))$, where the entries $\lambda$ and $\mu$ stand for $\lambda I_{H}$ and $\mu I_{H}$ and $S^{*}$ is the adjoint of $S$ in $B(H)$. Then $\mathcal{S}(V)$ is an operator system in $B\left(H^{2}\right)$. Let $I(\mathcal{S}(V))$ be the injective envelope of $\mathcal{S}(V)$ in $B\left(H^{2}\right)$ and let $\Phi: B\left(H^{2}\right) \rightarrow$ $I(\mathcal{S}(V))$ be a unital completely contractive onto projection. Hamana showed that $I(\mathcal{S}(V))$ has a $2 \times 2$-matrix such that 1-2 corner is the injective envelope $I(V)$ of $V$.

Lemma 2.3. Let $V$ be an operator space. Then $V$ is globally exact if and only if $\mathcal{S}(V)$ is globally exact.

Proof. $(\Leftarrow)$ Let $\mathcal{S}(V)$ be a globally exact operator space. As $V$ is $1-2$ corner subspace of $\mathcal{S}(V), V$ is also globally exact.

$(\Rightarrow)$ Let $W$ be a finite dimensional subspace of $\mathcal{S}(V)$. There is a finite dimensional subspace $W^{\prime} \subseteq V$ such that $W \subseteq \mathcal{S}\left(W^{\prime}\right)$. As $V$ is global exact, by Theorem 2.2, $I\left(W^{\prime}\right)$ is finite dimensional. By [25], $I\left(W^{\prime}\right)$ is completely isometric to $\bigoplus_{i=1}^{n} B\left(\mathbb{C}^{p_{i}}, \mathbb{C}^{q_{i}}\right)$, for some positive integer $n$. By $[18$, Theorem 2.2], $I\left(S\left(W^{\prime}\right)\right)$ is finite dimensional and so is $I(W)$. That means that $\mathcal{S}(V)$ is a globally exact operator space.

Theorem 2.4. Let $A$ be a unital $C^{*}$-algebra. Then $A$ is globally exact if and only if $A$ is finite dimensional.

Proof. A finite dimensional $C^{*}$-algebra is completely isometric to a finite direct sum $\bigoplus_{i=1}^{n} M_{m_{i}}(\mathbb{C})$, which is globally exact.

Conversely, let $A \subseteq B(H)$ be an infinite dimensional unital $C^{*}$-algebra. Then a maximal abelian self-adjoint unital subalgebra $C(\Omega)$ of $A$ is infinite dimensional ([26, Exercise I.11.1]). By induction, there is an infinite sequence $\left\{f_{n}\right\}_{n=1}^{\infty} \subseteq C(\Omega)$, with disjoint supports, and points $x_{n} \in \Omega$ such that $0 \leq$ $f_{n} \leq 1$ and $f_{n}\left(x_{n}\right)=1$. Let $f=\sum_{n=1}^{\infty} \frac{f_{n}}{n}$ and let $V$ be the finite dimensional subspace of $C(\Omega)$ generated by $1, f$ and $f^{2}$. Consider the corresponding completely contractive projection $\varphi: B(H) \rightarrow I(V)$, which is also unital, and 
thus completely positive. Let $C_{e}^{*}(V)$ be the corresponding $C^{*}$-subalgebra of $I(V)$ with the Choi-Effros product ([10, Theorem 6.1.3]). Then

$$
\varphi\left(f^{*} f\right)=\varphi\left(f^{2}\right)=f^{2}=f^{*} f=\varphi(f)^{*} \varphi(f)
$$

and by [10, Corollary 5.2.2], $\varphi(T) \varphi(f)=\varphi(T f)$, for any $T \in B(H)$. In particular,

$$
f^{3}=f^{2} f=\varphi\left(f^{2}\right) \varphi(f)=\varphi\left(f^{2} f\right)=f^{2} \circ_{\varphi} f \in C_{e}^{*}(V) .
$$

By induction, $f^{n+m}=f^{n} \circ_{\varphi} f^{m} \in C_{e}^{*}(V)$, for any $n, m \in \mathbb{N}$, that is, the product in $C_{e}^{*}(V)$ is the same as that of $A$. Thus $C_{e}^{*}(V)$ is the $C^{*}$-algebra generated by $V$ in $A$. On the other hand, if $\sum_{k=1}^{m} \alpha_{k} f^{k}=0$, then

$$
\sum_{k=1}^{m} \alpha_{k} f^{k}\left(x_{n}\right)=\sum_{k=1}^{m} \alpha_{k}\left(\frac{f_{n}}{n}\right)^{k}\left(x_{n}\right)=\sum_{k=1}^{m} \frac{\alpha_{k}}{n^{k}}=0,
$$

for each $n$, thus $\alpha_{k}=0$, for each $1 \leq k \leq m$, namely, $\left\{f^{k}\right\}_{k=1}^{\infty}$ is linearly independent in $C^{*}(V)$. Thus $C_{e}^{*}(V)=C^{*}(V)$ is not finite dimensional, and so is $I(V)$, thus $A$ is not globally exact by Theorem 2.2 .

Theorem 2.5. For Hilbert spaces $H$ and $K, B(H, K)$ is globally exact if and only if $H$ or $K$ is finite dimensional.

Proof. Let $B(H, K)$ be globally exact. Without loss of generality, we may assume that $\operatorname{dim} H \leq \operatorname{dim} K$, which let us to embed $B(H)$ completely isometrically into $B(H, K)$. Hence by Theorem $2.4, H$ is finite dimensional. The converse follows from a result of H. Rosenthal [23, Proposition 2.11].

By [24, Theorem 14.4.1] and [12, Remark 5.3], $C_{r}^{*}\left(\mathbb{F}_{n}\right)$ is a untial infinite dimensional exact $C^{*}$-algebra, for any $n \in \mathbb{N}, n \geq 2$ and for $n=\infty$. By Theorem 1.6, $C_{r}^{*}\left(\mathbb{F}_{n}\right)$ is not globally exact. This provides an example of a $C^{*}$-algebra which is uniformly exact in the sense of Rosenthal $([23])$, but not globally exact.

Next, let us give an example of a globally exact operator space $V$ such that $I(V)$ is not globally exact. Let $H$ be an infinite dimensional separable Hilbert space with orthonormal basis $\left\{e_{n}\right\}_{n=1}^{\infty}$. Embed the column and row Hilbert spaces $H_{c}$ and $H_{r}$ completely isometrically as the first column and row operator subspaces of $B(H)=M_{\infty}(\mathbb{C})$, for more detail see [10, Section 3.4]. Let $V$ be the operator space spanned by $H_{c}$ and $H_{r}$ in $B(H)$. Let $W$ be a finite dimensional operator subspace of $V$ and $p$ be the rank-1 projection $h \mapsto\left\langle h, e_{1}\right\rangle e_{1}$ in $B(H)$. Put $K_{1}=\left\{h \in H:(h)_{c} \in W p\right\}$ and $K_{2}=\{g \in H$ : $\left.(g)_{r} \in p W\right\}$. These are finite dimensional Hilbert spaces. Let $K$ be the finite dimensional Hilbert space spanned by $K_{1}$ and $K_{2}$ in $H$. We may regard $W$ as an operator subspace of $B(K)$. Since $B(K)$ is a finite dimensional injective operator space, $I(W) \subseteq B(K)$ is finite dimensional. Thus $V$ is globally exact. 
On the other hand, embed

$$
E=\left(\begin{array}{ll}
\mathbb{C} & \mathbb{C} \\
\mathbb{C} & 0
\end{array}\right)
$$

to a top left corner subspace in $V$, completely isometrically. Then, $I(E)=$ $M_{2}(\mathbb{C})$, which is the top left corner of $I(V)$. By induction, compact operators $K(H)$ sits as a subspace in $I(V)$, thus $I(V)=B(H)([4])$, which is not globally exact by Theorem 2.4.

Recall that an operator space $V$ is reflexive if $V^{* *} \cong V$, and locally reflexive if for any finite dimensional operator subspace $E \subseteq V^{* *}$, there is a net $\varphi_{\alpha}: V^{* *} \rightarrow V$ of completely contractive maps, whose restrictions to $V$ are point-weak ${ }^{*}$-convergent to the identity map on $V([10,14.3])$. It is known that exactness implies local reflexivity ([10, Corollary 14.6.5]).

Theorem 2.6. Let $V$ be an injective operator space. Then $V$ is globally exact if and only if it is reflexive.

Proof. Assume that $V$ is reflexive. It is well known that $\ell^{\infty}$ is not reflexive hence, by [5, Corollary 4.3], it could not be embedded completely isomorphically into $V$. By [1, Theorem 2.5], $V$ is completely isometric to a finite direct sum $\bigoplus_{i=1}^{n} B\left(K_{i}, H_{i}\right)$, such that each $H_{i}$ or $K_{i}$ is finite dimensional. By Theorem 2.5, $V$ is globally exact.

Conversely, assume that $V$ is globally exact. By Theorem $2.4, \ell^{\infty}$ is not globally exact and so can not be embedded completely isomorphically into $V$. Again by [1, Theorem 2.5], $V$ is completely isometric to a direct sum $\bigoplus_{i=1}^{n} B\left(K_{i}, H_{i}\right)$ with each $H_{i}$ or $K_{i}$ is finite dimensional. Therefore,

$$
\begin{aligned}
V^{* *} & \cong\left(\bigoplus_{i=1}^{n} B\left(K_{i}, H_{i}\right)\right)^{* *} \cong \bigoplus_{i=1}^{n} B\left(K_{i}, H_{i}\right)^{* *} \\
& =\bigoplus_{i=1}^{n} K\left(K_{i}, H_{i}\right)^{* *} \cong \bigoplus_{i=1}^{n} B\left(K_{i}, H_{i}\right) \cong V
\end{aligned}
$$

\section{ACKNOWLEDGEMENTS.}

The authors would like to thank the antonymous referee for the comments and corrections.

\section{REFERENCES}

[1] M. Amini, A. R. Medghalchi and H. Nikpey, On tensor products of injective operator spaces, Houston J. Math. 43 (2017), 1147-1163.

[2] R. Archbold and C. Batty, $C^{*}$-tensor norms and slice maps, J. London Math. Soc. (2) 22 (1980), 127-138. 
[3] D. Blecher and C. Le Merdy, Operator algebras and their modules-an operator space approach, London Mathematical Society Monographs, New Series 30, Oxford University Press, Oxford, 2004.

[4] D. Blecher and V. I. Paulsen, Multipliers of operator spaces and the injective envelope, Pacific J. Math. 200 (2001), 1-17.

[5] J. B. Conway, A course in functional analysis, Springer-Verlag, Berlin, 1990.

[6] Z. Dong and Z.-J. Ruan, Weak* exactness for dual operator spaces, J. Funct. Anal. 253 (2007), 373-397.

[7] E. G. Effros and U. Haagerup, Lifting problems and local reflexivity for $C^{*}$-algebras, Duke Math. J. 52 (1985), 103-128.

[8] E. G. Effros, M. Junge and Z.-J. Ruan, Integral mappings and the principle of local reflexivity for noncommutative $L^{1}$-spaces, Ann. of Math. (2) 151 (2000), 59-92.

[9] E. G. Effros, N. Ozawa and Z.-J. Ruan, On injectivity and nuclearity for operator spaces, Duke Math. J. 110 (2001), 489-521.

[10] E. Effros and Z.-J. Ruan, Operator spaces, Oxford University Press, New York, 2000.

[11] E. G. Effros and Z.-J. Ruan, On the abstract characterization of operator spaces, Proc. Amer. Math. Soc. 119 (1993), 579-584.

[12] U. Haagerup, Quasi traces on exact $C^{*}$-algebra are traces, Math. Reports of the Academy of Science of the Royal Society of Canada, arXiv:1403.7653.

[13] M. Hamana, Injective envelopes of operator systems, Publ. RIMS, Kyoto Univ. 15 (1979), 773-785.

[14] M. Hamana, Injective envelopes of dynamical systems, in: Operator algebras and operator theory, Longman, Harlow, 1992, 69-77.

[15] E. Kirchberg, The Fubini theorem for exact $C^{*}$-algebras, J. Operator Theory 10 (1983), 3-8.

[16] E. Kirchberg, Exact $C^{*}$-algebras, tensor products, and the classification of purely infinite algebras, in: Proceedings of the International Congress of Mathematicians, vols. 1, 2, Zurich, 1994, Birkhäuser, Basel, 1995, 943-954.

[17] E. Kirchberg, On subalgebras of the CAR-algebra, J. Funct. Anal. 129 (1995), 35-63.

[18] A. R. Medghalchi and H. Nikpey, Characterizing injective operator space $V$ for which $I_{11}(V) \cong B(H)$, Publ. Math. Debrecen 82 (2013), 21-30.

[19] G. Pisier, Exact operator spaces, in: Recent advances in operator algebras, Orlèan, 1992, Astèrisque 232 (1995), 159-186.

[20] G. Pisier, Introduction to operator space theory, Cambridge Univ. Press, Cambridge, 2003.

[21] A. R. Robertson, Injective matricial Hilbert spaces, Math. Proc. Cambridge Philos. Soc. 110 (1991), 183-190.

[22] A. G. Robertson and S. Wassermann, Completely bounded isomorphism of injective operator systems, Bull. London Math. Soc. 21 (1989), 285-290.

[23] H. Rosenthal, The complete separable extension property, J. Operator Theory 43 (2000), 329-374.

[24] Z.-J. Ruan, Injectivity of operator spaces, Trans. Amer. Math. Soc. 315 (1989), 89104.

[25] R. R. Smith, Finite dimensional injective operator spaces, Proc. Amer. Math. Soc. 128 (2000), 3461-3462.

[26] M. Takesaki, Theory of operator algebras. I, Springer, New York, 2002.

[27] S. Wassermann, Exact $C^{*}$-algebras and related topics, Seoul National University, Seoul, 1994.

[28] G. Wittstock, Extension of completely bounded $C^{*}$-module homomorphisms, in: Operator algebras and group representations, Vol. II, Pitman, Boston, 1984, 238-250. 
M. Amini

Department of Mathematics

Tarbiat Modares University

Tehran 14115-134

Iran

and

School of Mathematics

Institute for Research in Fundamental Sciences (IPM)

Tehran 19395-5746

Iran

E-mail: mamini@modares.ac.ir, mamini@ipm.ir

A. R. Medghalchi

Department of Mathematics

Kharazmi University

50 Taleghani Avenue, Tehran 15618

Iran

E-mail: a_medghalchi@khu.ac.ir

H. Nikpey

Department of Mathematics

Shahid Rajaei Teacher Training University

Tehran 16785-136

Iran

E-mail: hamednikpey@gmail.com

Received: 29.1.2017.

Revised: 21.9.2017. 